\title{
Comparison of parallel or convergent proximal schanz screw placement of pertrochanteric fixator in intertrochanteric fracture model
}

\author{
Arif Gok ${ }^{1}$, Sermet Inal ${ }^{2}$, Ferruh Taspinar ${ }^{3}$, Eyyup Gulbandilar ${ }^{4}$, and Kadir Gok ${ }^{5}$ \\ ${ }^{1}$ Amasya University, Technology Faculty, Mechanical Engineering, 05000 Amasya, Turkey \\ ${ }^{2}$ Dumlupinar University, School of Medicine, Department of Orthopaedics and Traumatology, \\ Campus of Evliya Celebi, 43100 Kutahya, Turkey \\ ${ }^{3}$ Dumlupinar University, School of Health Science, Department of Physiotherapy and Rehabilitation, \\ 43100 Kutahya, Turkey \\ ${ }^{4}$ Eskisehir Osmangazi University, Faculty of Engineering \& Architecture, Department of Computer \\ Engineering, Meselik Campus, 26480 Eskisehir, Turkey \\ ${ }^{5}$ Manisa Celal Bayar University, Hasan Ferdi Turgutlu Teknoloji Fakültesi, Mechanical and Manufacturing \\ Engineering, 45400 Manisa, Turkey \\ Correspondence to: Arif Gok (arif.gok@amasya.edu.tr)
}

Received: 20 February 2017 - Accepted: 15 May 2017 - Published: 15 August 2017

\begin{abstract}
Intertrochanteric femoral fractures are serious traumas among elderly patients. In these patients, external fixator is a preferable method for the fixation of fractures. Therefore, this study was planned to compare the parallel and convergent proximal schanz screw placement of pertrochanteric fixator in the intertrochanteric femoral fractures with respect to biomechanical forces that stabilize the fracture line and to present their clinical importance. A commercial finite element based program, AnsysWorkbench was used to investigate the biomechanical parameters of the femoral intertrochanteric fractures and different placement of implants. The von Mises stress, von Mises strain and shear stress on the proximal and distal surface of the fracture line were lower in the convergent pertrochanteric fixator. Proximal schanz screws in convergent configuration pertrochanteric fixator had greater stress and strain values than proximal schanz screws in parallel configuration pertrochanteric fixator. The distance between the proximal schanz screws on the fracture line was measured as $12 \mathrm{~mm}$ in convergent configuration pertrochanteric fixator, and as $3.5 \mathrm{~mm}$ in parallel configuration pertrochanteric fixator. The angle between the proximal schanz screws in the convergent configuration was measured as $12.88^{\circ}$. The effect of convergent and parallel configuration pertrochanteric fixators on axial loading demonstrated that convergent configuration pertrochanteric fixator was safer in this respect.
\end{abstract}

\section{Introduction}

Intertrochanteric femoral fractures (ITFs) are generally associated with low energy traumas among elderly patients and with high energy traumas among young patients. Especially in elderly patients, and depending on age-related concomitant conditions such as osteoporosis, these fractures can lead to high morbidity and mortality rates. For this reason, ITFs are considered among the difficult-to-treat group of fractures (Healey and Msorman, 1993; Laskin et al., 1979). These fractures occur especially among patients with a history of diabetes, heart disease or chronic obstructive pulmonary disease. For elderly patients with these concurrent conditions, the administration as well as the duration of anesthesia involves certain risks (Christodoulou and Sdrenias, 2000). For this reason, it is generally described that treatment procedures involving external fixation methods should be preferred for such patients. External fixation methods ensure treatment with minimal surgical trauma and blood loss, and a short duration of anesthesia and surgery (Dhal and Singh, 

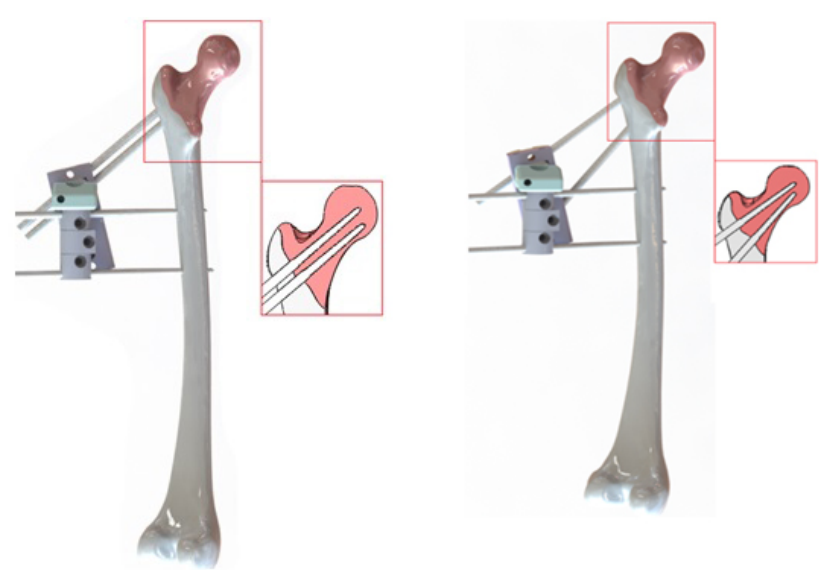

Figure 1. Images of PTF with different configuration.

1996; Kamble et al., 1996; Moroni et al., 2005; Vossinakis and Badras, 2001, 2002a). Earlier mobilization of patients allows the prevention of postoperative complications such as urinary infections, pneumonia, decubitus ulcers and bone infections (Badras et al., 1997; Eksioglu et al., 2000).

External fixators have been used since 1950. However, despite the limited number of patients treated with external fixators in the literature, complications such as pin site infections, varus deformities and shortness have been described (Barros et al., 1995; Christodoulou and Sdrenias, 2000; Kamble et al., 1996). To ensure early rehabilitation with these fractures, the most important goal is to preserve the reduction position of the fracture (Irfan, 1997; Pervez et al., 2004; Vossinakis and Badras, 2002b). For this reason, the effectiveness of external fixators on fracture stability should be considered as a key point. Aside from comparing the stabilizing effect of different external fixators applied together with other internal fixation materials, it is also necessary to investigate the effectiveness of different implantation configurations. Thus, by identifying which configurations would ensure stronger stabilization by fixators implanted for hip fractures, it becomes possible to predict the quality of mobilization. As a result, possible complications can be prevented. In our study, our aim was biomechanically to investigate the effectiveness of Pertrochanteric Fixators (PTFs) applied with two different configurations of schanz screws inserted to the femoral head in ITFs.

\section{Materials and methods}

\section{Modeling using Finite Element Method (FEM) of the femur}

A commercial finite element based program, MSC Patran/Mentat/Marc is used to investigate the biomechanical parameters of the femoral fracture and implants (Mahaisavariya et al., 2006). The human femoral model is scanned using 3 dimension (3-D) scanner and point cloud is obtained. After that, 3-D model of femur is created using

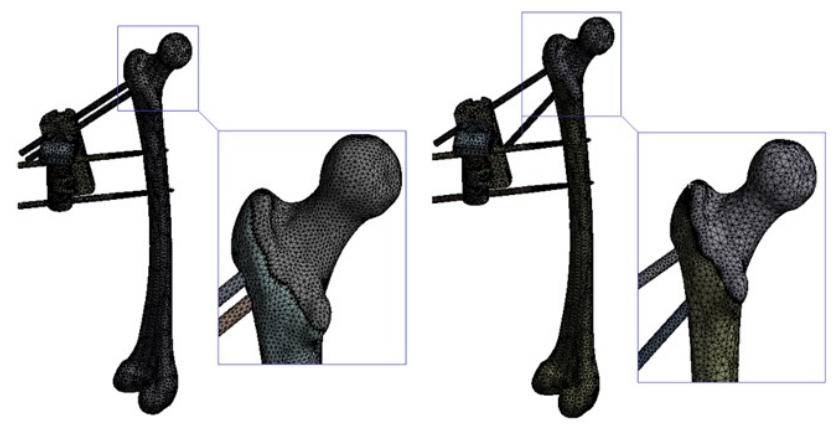

Figure 2. Mesh structure of the model.

Table 1. Biomechanical properties of the femur.

\begin{tabular}{lr}
\hline Parameters & Value \\
\hline Density $\left(\mathrm{kg} \mathrm{m}^{-3}\right)$ & 2100 \\
Young's modulus (GPa) & 17 \\
Yielding strength (MPa) & 135 \\
Tensile strength (MPa) & 148 \\
Poisson's ratio & 0.35 \\
\hline
\end{tabular}

point cloud data by Geomagic Studio 10 programme. The femoral ITF is created using SolidWorks programme as seen in Fig. 1. The modeling of the implants are also modeled in 3D using the SolidWorks 2013 programme. These models are imported into the AnsysWorkbench to prepare the FEM and the mesh generation of the FEM is created. The mesh generation of the models is created using the tetrahedrons element type as seen in Fig. 2. The generated finite element model has 147783 nodes and 96290 elements. While the element size of the model is selected as $4 \mathrm{~mm}$, the contact regions are selected as $2 \mathrm{~mm}$.

The femur is fixed from the distal condylar articular face. The Pertrochanteric Fixator (PTF) implantations are performed similar to the surgical implantation technique used in routine orthopaedic surgeries. Two proximal schanz screws of fixators are applied in two different configurations, the first one is performed in parallel and the second one is applied in convergent way (Fig. 1). The distal schanz screws of both PTFs are applied in the same configuration; perpendicular to the femoral shaft and parallel to each other. Contact types among the parts of the implants and bone are defined as a frictional contact. These contact interactions are assumed between the different parts of the models. Friction coef?cients are taken as 0.46 for bone-bone interactions and 0.42 for bone-implant interactions. For boundary conditions, the loading vector ( $350 \mathrm{~N}$ by $z$-axis) is applied through the orthogonal plane to the femoral head while the distal end of the femur is fixed (Goffin et al., 2013). The FEM is applied to both PTF configurations. All materials used for PTF were stainless steel from the ANSYS Material Library. The biomechanical properties are summarized in Table 1 (Tu et al., 


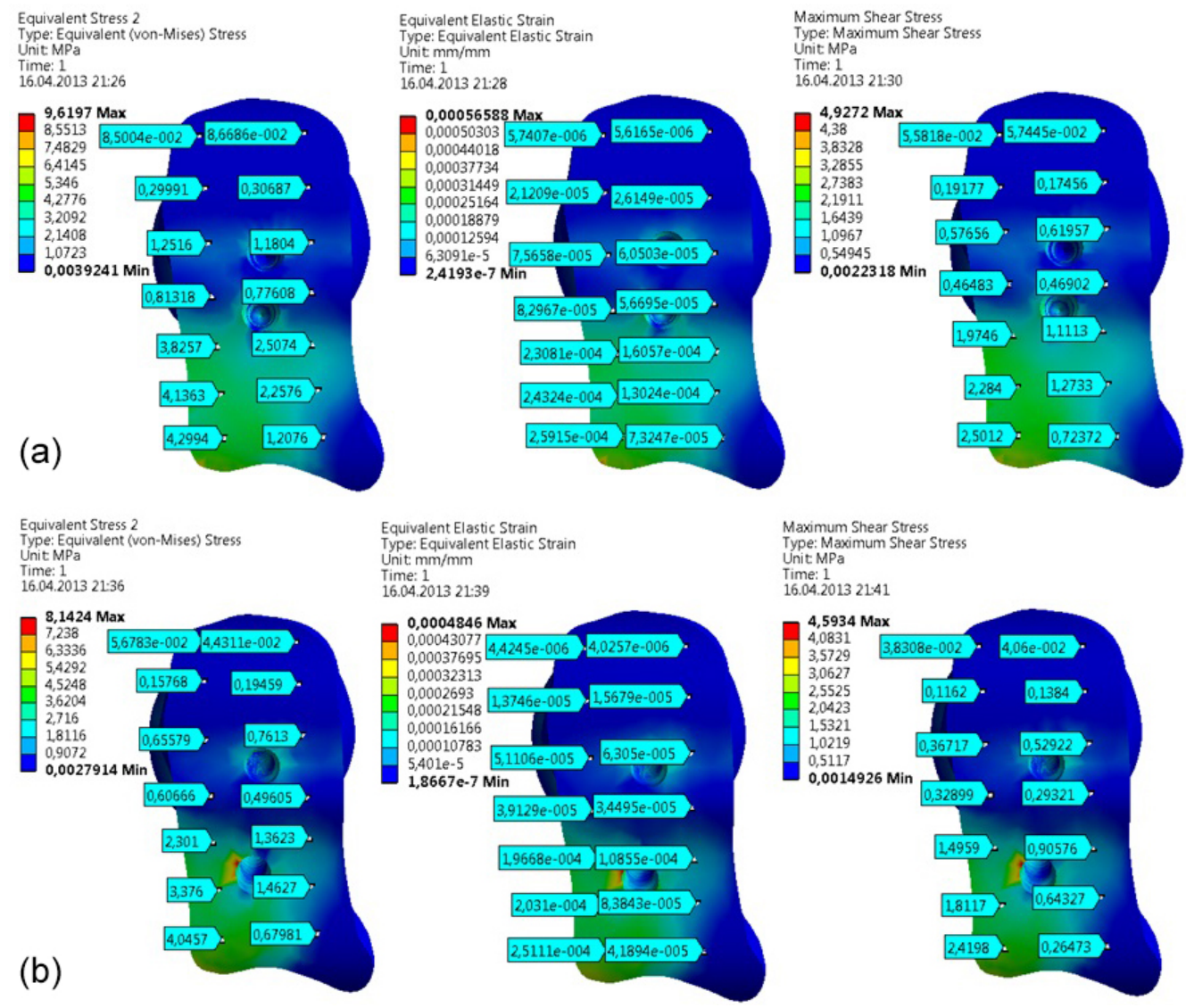

Figure 3. The biomechanical parameters on the proximal surface of the fracture line for both configurations.

2009). After the boundary conditions, biomechanical properties of the materials for each component were identified and simulations were solved. The Von-Mises stress, strain, shear stress values are evaluated at the upper and lower surfaces of the fracture line and implants for each configuration. Also the distances between the proximal schanz screws at the fracture line for each configuration are measured. The angle between proximal schanz screws in convergent technique is also evaluated. The parameters are compared and the research question to this study was whether different proximal schanz screw placement of PTFs might have biomechanical advantages during axial loading for patients to be mobilized.

\section{Results}

The Von Mises stress, strain and shear stress values on the upper and lower surfaces of the fracture line were found to be lower in convergent configuration PTF in comparison to parallel configuration PTF (Figs. 3 and 4). It was also determined that proximal schanz screws in convergent configuration PTF had greater stress and strain values than proximal schanz screws in parallel configuration PTF (Figs. 5 and 6). The distance between the proximal schanz screws on the fracture line was measured as $12 \mathrm{~mm}$ in convergent con- figuration PTF, and as $3.5 \mathrm{~mm}$ in parallel configuration PTF. The angle between the proximal schanz screws in the convergent configuration was measured as $12.88^{\circ}$ (Table 2).

\section{Discussion}

For the past 40 years, through various cadaver studies, model and clinical trials, researchers have investigated new materials and techniques that would ensure early and safe rigid fixation and load transfer (Audigé et al., 2003; Bong et al., 2004; Bridle et al., 1991). Studies in recent years have demonstrated that, in comparison to past studies, better results are now being obtained with external fixators used for the treatment of trochanteric fractures (Christodoulou and Sdrenias, 2000; Vossinakis and Badras, 2001, 2002a). This study was performed in order to compare the biomechanical effects of convergent and parallel configuration PTFs during axial loading by using FEM. It was demonstrated in this context that PTF with convergent configuration ensured better stabilization of the fracture line.

An evaluation of the literature revealed no studies on the biomechanical properties of external fixators used in different configurations for hip fractures, and the effect of these configurations on clinical outcome. The majority of the stud- 
Table 2. The biomechanical parameters at the fracture line.

\begin{tabular}{lrr}
\hline Variables & Parallel & Convergent \\
\hline Von Mises stress & $1.64 \pm 1.5$ & $1.14 \pm 1.24$ \\
The proximal surface & $0.80 \pm 0.7$ & $0.68 \pm 0.61$ \\
The distal surface & $10.1 \pm 8.6$ & $7.9 \pm 8$ \\
\hline Von Mises strain & $4.6 \pm 3.9$ & $4.5 \pm 3.18$ \\
The proximal surface $\left(10^{-5}\right)$ & $0.88 \pm 0.82$ & $0.66 \pm 0.73$ \\
The distal surface $\left(10^{-5}\right)$ & $0.49 \pm 0.43$ & $0.40 \pm 0.33$ \\
\hline Shear & 65.915 & 68.35 \\
The proximal surface $\left(10^{-5}\right)$ & 0.00034 & 0.00035 \\
The distal surface $\left(10^{-5}\right)$ & 0 & 12.88 \\
\hline Maximum Von Mises stress (schanz) & 3.5 & 12 \\
\hline Maximum Von Mises strain (schanz) & & \\
\hline The angle between proximal schanz screws (degree) & & \\
\hline The distance between the proximal schanz screws on the fracture line $(\mathrm{mm})^{*}$ & & \\
\hline
\end{tabular}

* mm: milimetres.
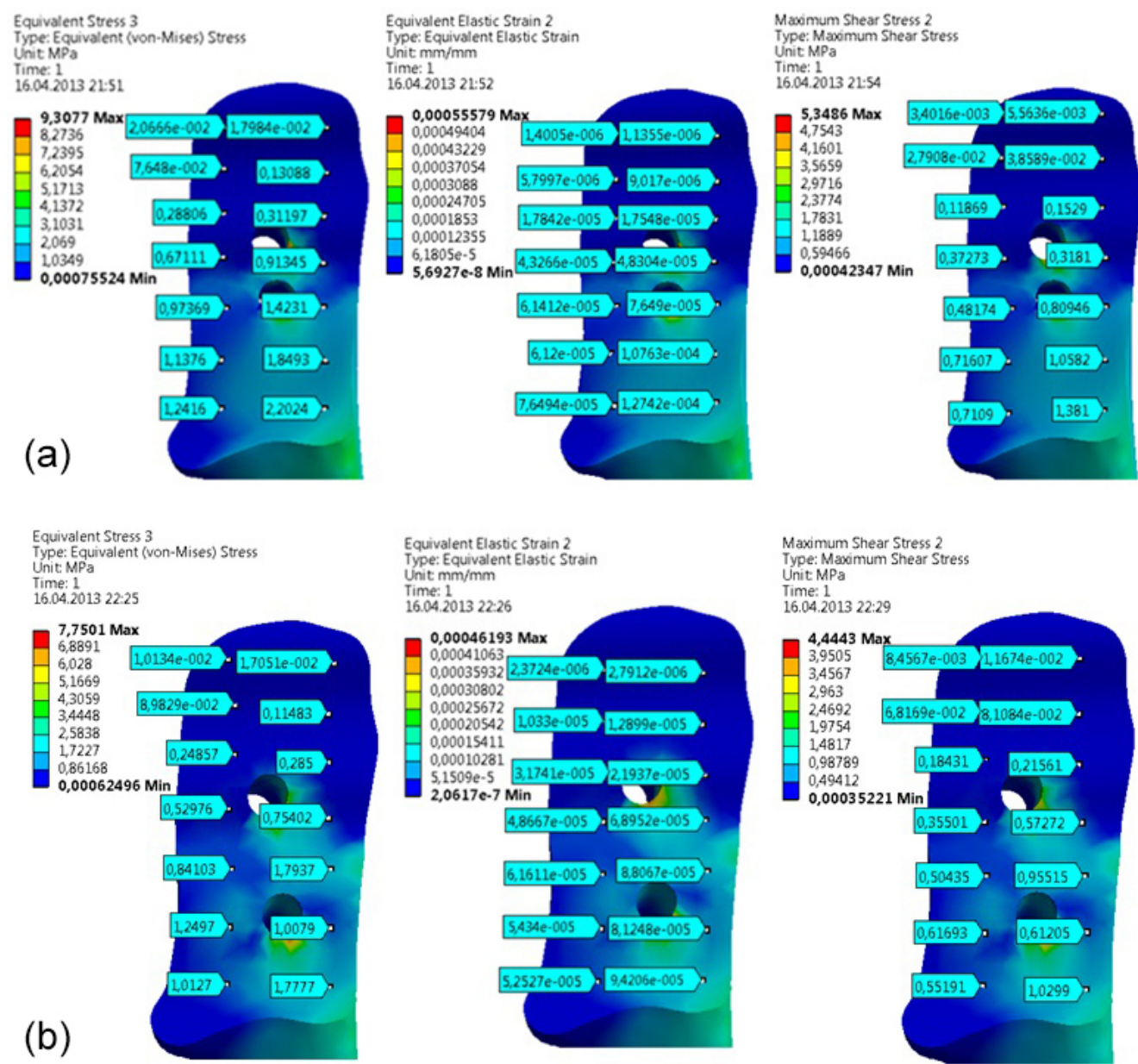

Figure 4. The biomechanical parameters on the distal surface of the fracture line for both configurations. 

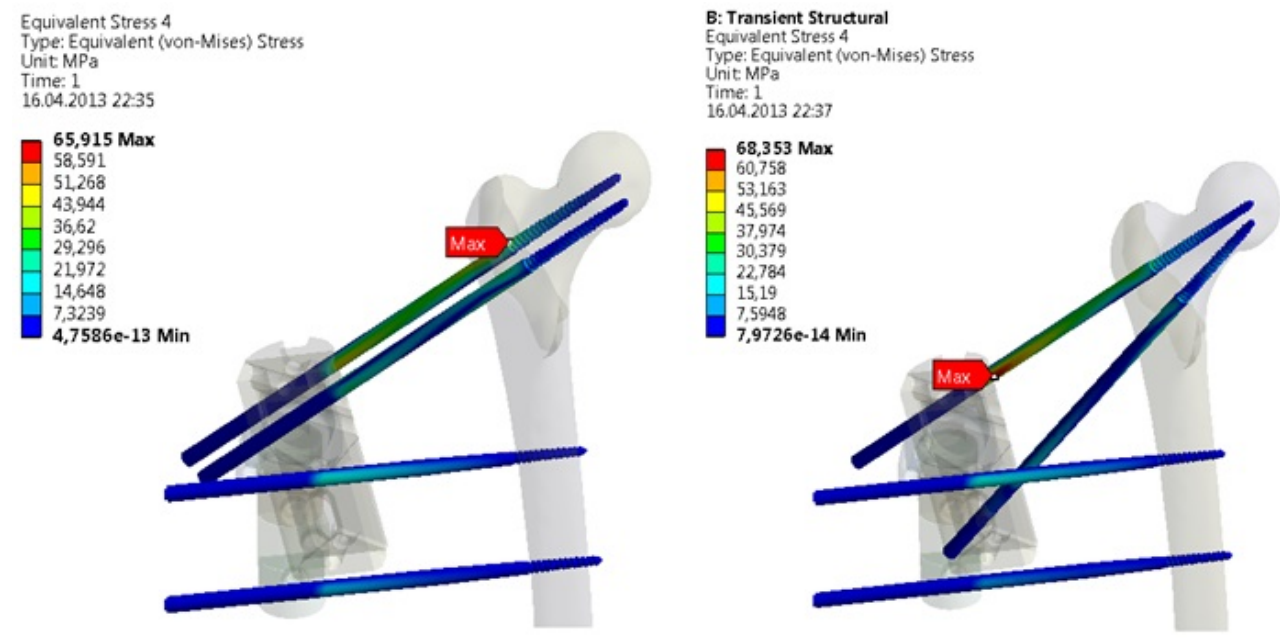

Figure 5. Demonstration of the maximum stress of proximal schanz screws.
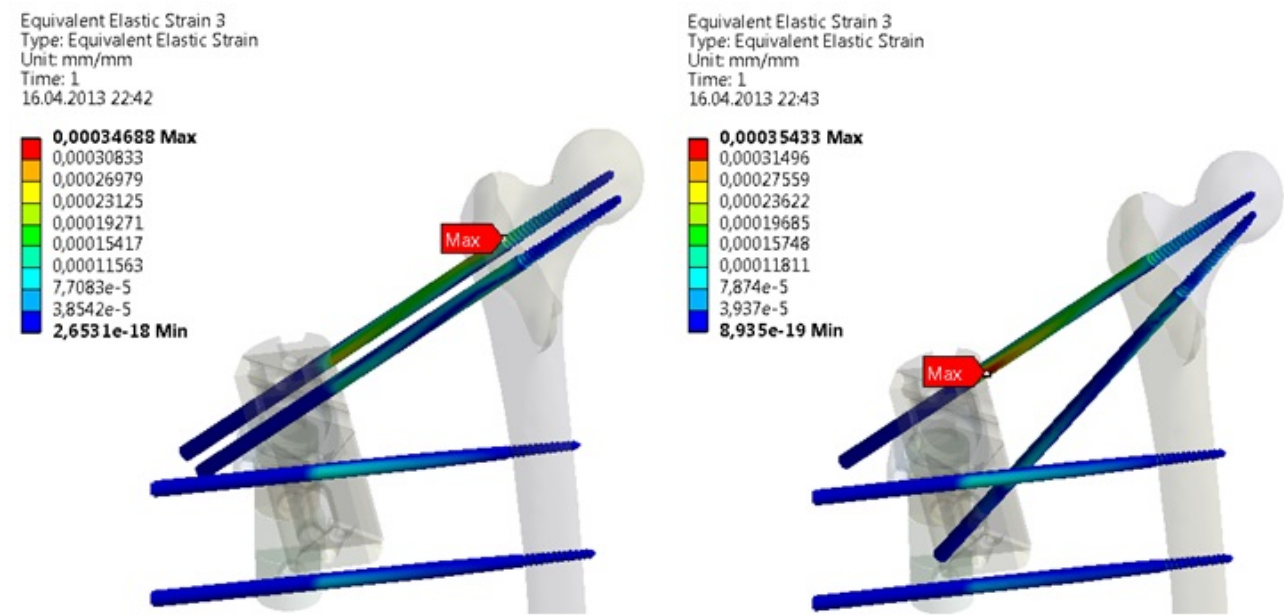

Figure 6. Demonstration of the maximum strain of proximal schanz screws.

ies on external fixators used for hip fractures demonstrate minimal blood loss, reduced surgery risk and earlier mobilization for this treatment method. They also show a very low incidence of postoperative complications, and a low rate of morbidity and mortality associated with these complications (Kazakos et al., 2007; Ozkaya et al., 2008).

Vekris et al. (2011) clinically compared parallel and convergent configured PTFs used for the treatment of ITFs. Based on the study results, they suggested that parallel and convergent configured PFTs provided the same clinical results, and that the parallel configuration was preferable due to its easier applicability. No biomechanical analyses were conducted in this study; only a clinical interpretation of the results was performed.

In the study of Eksioglu et al. (2000), it was described that internal fixators were insufficient for early rehabilitation, and that they did not allow walking with full weight bearing until union occurs. In addition to this, they emphasized that delayed and difficult rehabilitation was associated with an increase in early mortality rates. For this reason, Eksioglu reported that external fixators applied for ITFs should be considered as semi-conservative method. In recent years, the increase in the number of studies performed on external fixators has led the authors of this article to biomechanically investigate different configurations that can be applied with external fixators. To our knowledge, there is currently no other biomechanical research in the literature regarding this subject.

As lateral fixation devices lead to compression in larger bones, they also tend to reduce the shear forces between surfaces (Scarante et al., 1993). Closed reduction protects the fracture hematoma and ensures rapid healing (Vossinakis and Badras, 2002b). In patients with trochanteric fractures they treated with external fixators, Aly et al. (2004) described that 
external fixation provided good protection for the reduction, and also corrected the varus deformity while causing compression on the fracture line. In addition to this, although certain mechanical problems have been reported with the external fixators, it was described that the elasticity of the schanz screws and the effects of the tension band supported the mechanical stability (Vossinakis and Badras, 2002a). In the study of Özdemir et al. (2003), it was described that union in the treatment of trochanteric fractures with external fixators required an average of 10.9 weeks. However, this recovery period was identified in elderly and high risk patients; it is hence possible to consider and plan studies involving young patients and different configurations. The ability to transfer with full weight bearing at an earlier stage might potentially result in a shorter recovery period.

Moroni et al. (2005) investigated various screw properties in their study, and determined that external fixators with hydroxyapatite-coated screws could potentially be used for osteoporotic elderly patients. Certain researchers have also described that the schanz screws in external fixators could be used in different numbers and angles depending on the width of the femoral neck and the preferences of the surgeon (Girgin et al., 1993; Subaş1 et al., 1998). However, it should be considered that the biomechanical responses of the implants will also be different depending on the differences in application. As such, these biomechanical properties should be investigated and adapted for clinical use. Researchers will thus be able to have an opinion on the stabilization of the fracture line, and to plan and design further clinical studies accordingly.

Baumgaertner (Vossinakis and Badras, 2002b) described that fracture fixation is of great importance for bone healing. In our study, we observed that the convergent configuration had lower stress, strain and shear stress values on the upper and lower surface of the fracture line in comparison to the parallel configuration. Furthermore, it was observed that the stress and strain in the proximal schanz screws were greater in the convergent configuration. This demonstrated that convergent configuration PTF assumed a greater portion of the loads on the fracture line, and that they hence provided greater stability. On the other hand, these same biomechanical parameters were observed as being lower in schanz screws in parallel configuration PTF. This confirmed that the parallel configuration bore less load and hence assumed a smaller portion of the loads on the fracture line. Although Vekris et al. (2011) described the parallel configuration as a preferable method in terms of its ease of applicability, they were not able to biomechanically demonstrate this configuration's resistance against axial loading. The conclusion we reached with regards to biomechanics was different from the one reached by Vekris et al. (2011) in their clinical study.

Especially in severe osteoporotic patients, we believe that it is necessary to investigate the different configurations of external fixators in order to prevent and overcome mechanical complications such as the shortness of extremity and varus deformity. This is because these patients are generally elderly patients with poor overall health and bone quality. For this reason, positive differences that can be biomechanically produced and proposed are of considerable importance. This will assist in the planning of the ideal configuration and treatment method for the patient.

There are various stability studies describing the use of diagonal or parallel Kirschner wires in the surgical treatment of elbow fractures. Tachdjian (John Anthony, 2002) describes two different techniques for supracondylar elbow fractures. One of these techniques involves the application of the wires on the fracture line in a diagonal configuration, while second technique involves the application of the wires in a parallel configuration. Furthermore, as an aspect that is more important than the diagonal or parallel application of the wires, it was described that increasing the distance between the Kirschner wires passing over the fracture line led to greater stability. In our study, the distance between the schanz screws over the fracture line was 3.5 times greater in the convergent configuration in comparison to the parallel configuration. Our results are thus in parallel with Tachdjian's observations. We believe that placing the schanz screws sufficiently apart such that they remain inside the femur neck will also increase the distance between the pins, and thereby strengthen the stability. However, it should also be considered that increasing the angle will also increase the likelihood of mechanical complications during surgery. The elbow joint is not an articulation that bears as much direct load as the hip joint. We believe that it is very important to bear this consideration in mind, since the hip joint is constantly subject to axial loading and carries more weight than the elbow joint. According to our results, schanz screws placed convergently on a simple ITF ensured greater stabilization of the fracture line. For this reason, this configuration could be preferred to the parallel configuration in osteoporotic patients.

Although we identified studies in the literature in which the schanz screws of external fixators were applied at different angles to the femoral head of cases with ITFs, we nevertheless did not encounter any study describing the effects of applications at different angles on the biomechanical stability across the fracture line. For this reason, there is no clear information on which configuration is more advantageous. We also did not encounter any study similar to our own that biomechanically compared PTFs of different configurations. As it involved a biomechanical analysis performed by using FEM method (a method known to be reliable), our study was considered to be reliable and valid. However, problems such as the inability to perform three dimensional scans on the human femur, the assumption that the femur has an equally distributed density, the inability to show the proximal trabecular structure and the calcar, cortical, spongiform bony structures, and the inability to reflect the extent of osteoporosis (if applicable) represented the limitations of this study. 


\section{Conclusion}

An investigation of the effect of convergent and parallel configuration PTFs on axial loading demonstrated that convergent configuration PTF was safer in this respect. We believe that this observation will contribute to future clinical applications.

Data availability. All the data used in this manuscript can be obtained by requesting from the corresponding author.

Competing interests. The authors declare that they have no conflict of interest.

Edited by: D. Pisla

Reviewed by: M. B. Bilgin and D. Tarnita

\section{References}

Aly, T. A., Hafez, K., El-nor, T. A., and, Osama, A.: Treatment of Trochanteric Fractures By External Fixator in Patients With High Unacceptable Operative Risk, Pan Arab. J. Orth. Trauma, 8, 157162,2004

Audigé, L., Hanson, B., and Swiontkowski, M. F.: Implant-related complications in the treatment of unstable intertrochanteric fractures: meta-analysis of dynamic screw-plate versus dynamic screw-intramedullary nail devices, Int. Orthop., 27, 197-203, 2003.

Badras, L., Skretas, E., and Ed, V.: Traitement des fractures pertrochantériennes par fixateurexterne, Rev. Chir. Orthop., 84, 461-465, 1997.

Barros, J. W., Ferreira, C. D., Freitas, A. A., and Farah, S.: External fixation of intertrochanteric fractures of the femur, Int. Orthop., 19, 217-219, 1995.

Bong, M. R., Patel, V., Iesaka, K., Egol, K. A., Kummer, F. J., and Koval, K. J.: Comparison of a Sliding Hip Screw with a Trochanteric Lateral Support Plate to an Intramedullary Hip Screw for Fixation of Unstable Intertrochanteric Hip Fractures: A Cadaver Study, J. Trauma Acute Care Surg., 56, 791-794, 2004.

Bridle, S., Patel, A., Bircher, M., and Calvert, P.: Fixation of intertrochanteric fractures of the femur. A randomised prospective comparison of the gamma nail and the dynamic hip screw, J. Bone Joint Surg., 73-B, 330-334, 1991.

Christodoulou, N. A. and Sdrenias, C. V.: External Fixation of Select Intertrochanteric Fractures With Single Hip Screw, Clin. Orthop. Rel. Res., 381, 204-211, 2000.

Dhal, A. and Singh, S. S.: Biological fixation of subtrochanteric fractures by external fixation, Injury, 27, 723-731, 1996.

Eksioglu, F., Gudemez, E., Cavusoglu, T., and Sepici, B.: Treatment of intertrochanteric fractures by external fixation, Bull. Hosp. Joint. Dis., 59, 131-135, 2000.

Girgin, O., Öztan, L., Özlü, K., and Ög, B.: İntertrokanterik femur kırı klarının eksternal fiksatör ile tedavisi, 15-19 May 1993, Nevşehir, Türkiye, 607-610, 1993.

Goffin, J. M., Pankaj, P., and Simpson, A. H.: The importance of lag screw position for the stabilization of trochanteric fractures with a sliding hip screw: A subject-specific finite element study, J. Orthop. Res., 31, 596-600, 2013.

Healey, H. S. and Msorman, L.: Osteoporosis, in: Manual of Rheumatology and Outpatient Orthopaedic Disorders, Little Brown, Boston, 1993.

Irfan, Ö.: Kalça kırı klarında prognozu etkileyen risk faktörleri, Acta Orthop. Traumatol. Turc., 31, 374-377, 1997.

John Anthony, H.: Upper Extremity Injuries, in: Tachdjian's Pediatric Orthopaedics, W. B. Saunders Company, USA, 2002.

Kamble, K. T., Murthy, B. S., Pal, V., and Ráo, K. S.: External fixation in unstable intertrochanteric fractures of femur, Injury, 27 139-142, 1996.

Kazakos, K., Lyras, D. N., Verettas, D., Galanıs, V. P. I., and Kostas, X.: External fixation of intertrochanteric fractures in elderly high-risk patients, Acta Orthop. Belg., 73, 44-48, 2007.

Laskin, R. S., Gruber, M. A., and Zimmerman, A. J.: Intertrochanteric Fractures of the Hip in the Elderly: A Retrospective Analysis of 236 cases, Clin. Orthop. Rel. Res., 141, 188-195, 1979.

Mahaisavariya, B., Sitthiseripratip, K., and Suwanprateeb, J.: Finite element study of the proximal femur with retained trochanteric gamma nail and after removal of nail, Injury, 37, 778-785, 2006.

Moroni, A., Faldini, C., Pegreffi, F., Hoang-Kim, A., Vannini, F., and Giannini, S.: Dynamic Hip Screw Compared with External Fixation for Treatment of Osteoporotic Pertrochanteric Fractures, A Prospective, Random. Study, 87, 753-759, 2005.

Özdemir, H., Dabak, T. K., Ürgüden, M., and Gür, S.: A different treatment modality for trochanteric fractures of the femur in surgical high-risk patients: a clinical study of 44 patients with 21 month follow-up, Arch. Orthop. Trauma Surg., 123, 538-543, 2003.

Ozkaya, U., Parmaksızoglu, A. S., Gul, M., Kabukcuoglu, Y., Ozkazanl, G., and Basilgan, S.: Management of osteoporotic pertrochanteric fractures with external fixation in elderly patients, Acta Orthop. Traumatol. Turc., 42, 246-251, 2008.

Pervez, H., Parker, M. J., and Vowler, S.: Prediction of fixation failure after sliding hip screw fixation, Injury, 35, 994-998, 2004.

Scarante, B., Ranellucci, M., and Feas, L.: The dynamic axial fixator in the treatment of pertrochanteric fractures of the femur, Int J. Orthop. Trauma, 3, 58-60, 1993.

Subaşı, M., Atlıhan, D., Katırcı, T., Dindar, N., Aşık, Y., and Hasan, Y.: İntertrokanterik femur kırı klarının eksterna lfiksatör ile tedavisi, Acta Orthop. Traumatol. Turc., 32, 40-43, 1998.

Tu, Y. K., Liu, Y. C., Yang, W. J., Chen, L. W., Hong, Y. Y., Chen, Y. C., and Lin, L. C.: Temperature Rise Simulation During a Kirschner Pin Drilling in Bone, 11-13 June 2009, 3rd International Conference on Bioinformatics and Biomedical Engineering, ICBBE 2009, Beijing, China, 1-4, 2009.

Vekris, M. D., Lykissas, M. G., Manoudis, G., Mavrodontidis, A. N., Papageorgiou, C. D., Korompilias, A. V., Kostas-Agnantis, I. P., and Beris, A. E.: Proximal screws placement in intertrochanteric fractures treated with external fixation: comparison of two different techniques, J. Orthop. Surg. Res., 6, 48-60, 2011.

Vossinakis, I. C. and Badras, L.: Management of pertrochanteric fractures in high-risk patients with an external fixation, Int. Orthop., 25, 219-222, 2001. 
Vossinakis, I. C. and Badras, L. S.: The external fixator compared with the sliding hip screw for pertrochanteric fractures of the femur, J. Bone Joint Surg., 84-B, 23-29, 2002a.
Vossinakis, I. C. and Badras, L. S.: The Pertrochanteric External Fixator Reduced Pain, Hospital Stay, and Mechanical Complications in Comparison with the Sliding Hip Screw, J. Bone Joint Surg., 84, 1488-1501, 2002b. 